HD-THEP-02-24

\title{
Lagrange versus Symplectic Algorithm for Constrained Systems
}

\author{
Heinz J. Rothe f and Klaus D. Rothe f \\ Institut für Theoretische Physik - Universität Heidelberg \\ Philosophenweg 16, D-69120 Heidelberg, Germany
}

(August 2002)

\begin{abstract}
The systematization of the purely Lagrangean approach to constrained systems in the form of an algorithm involves the iterative construction of a generalized Hessian matrix $W$ taking a rectangular form. This Hessian will exhibit as many left zero-modes as there are Lagrangean constraints in the theory. We apply this approach to a general Lagrangean in the first order formulation and show how the seemingly overdetermined set of equations is solved for the velocities by suitably extending $W$ to a rectangular matrix. As a byproduct we thereby demonstrate the equivalence of the Lagrangean approach to the traditional Dirac-approach. By making use of this equivalence we show that a recently proposed symplectic algorithm does not necessarily reproduce the full constraint structure of the traditional Dirac algorithm.
\end{abstract}

\footnotetext{
${ }^{1}$ email: h.rothe@thphys.uni-heidelberg.de

${ }^{2}$ email: k.rothe@thphys.uni-heidelberg.de
} 


\section{Introduction}

A number of algorithms have been developed over the past years for treating constrained Hamiltonian systems. Perhaps the most familiar one to the physicist community is the one developed by Dirac [1]. Although very elegant and powerful in its algebraic structure, this algorithm has been critizized for being based on the existence of so called "primary constraints", which are a purely phase-space artefact, and have no counterpart on the Lagrangean level. Faddeev and Jackiw [2] have thus proposed an alternative method based on a first order Lagrangean (symplectic) formulation, avoiding the introduction of primariy constraints. Furthermore, the local symmetries of the Hamiltonian, as generated by the so called "first-class" constraints in Dirac's terminology, turn out to be larger than those of the Lagrangean This has led to a renewed interest in the problem of deducing the local symmetries of a Lagrangean from the Hamiltonian formalism [3], and in particular to a revival of the "Lagrangean approach", and the "symplectic approach" to constrained systems [4, 5, 6, 7, 8, 9]. Of all three methods, the Lagrangean algorithm is actually the most pedestrian one, with a solid mathematical basis. The symplectic algorithm, on the other hand, as developed in a series of elegant papers in refs. 脢, lacks a rigorous mathematical justification, and can lead, as we shall demonstrate, to an incomplete solution of the problem. In order to establish the relation between the three formalisms, we shall thus take as our starting point the Lagrangean approach as applied to first order Lagrangeans, in order to allow for a comparison with the symplectic approach. As shown in section 2, the Lagrangean algorithm leads to a larger set of equations than the number of unknown velocities to be solved for. This is reflected in the fact that the generalized Hessian which implements the algorithm, is a rectangular matrix possessing as many left zero-modes, as there are Lagrangean constraints hidden in the Euler-Lagrange equations. 
We show that these zero-modes are of such a form, that they permit the solution of the equations of motion in terms of the inverse of a quadratic matrix, whose elements are just the Poisson brackets of the Hamiltonian constraints - including the primary constraints. We thereby establish the equivalence with Dirac's algorithm. In section 3 we then consider the simple example of the particle motion on a hypersphere and thereby demonstrate that the symplectic algorithm of ref. [4] is not always equivalent to the Dirac and Lagrangean approach. We conclude this section by discussing the general condition under which this symplectic algorithm fails. Section 5 summarizes our findings.

\section{The Lagrangean algorithm}

Given a second order Lagrangean, one can always find an equivalent first order Lagrangean of the form

$$
L(Q, \dot{Q})=a_{\alpha}(Q) \dot{Q}_{\alpha}-V(Q)
$$

where $Q$ stands for $n$ degrees of freedom $Q_{\alpha}, \alpha=1,2 \cdots, n$. The corresponding Euler-Lagrange equations read

$$
W_{\alpha \beta}^{(0)}(Q) \dot{Q}_{\beta}=\frac{\partial V(Q)}{\partial Q_{\alpha}}
$$

where the matrix $W^{(0)}$ is defined by

$$
W_{\alpha \beta}^{(0)}(Q)=\frac{\partial a_{\beta}}{\partial Q_{\alpha}}-\frac{\partial a_{\alpha}}{\partial Q_{\beta}}
$$

Let $r_{0}$ be the rank of the matrix $W^{(0)}$. Then there exist $n-r_{0}$ zero modes of $W^{(0)}$, which we denote by $u^{(0)}(a), a=1 \cdots n-r_{0}$. Multiplying equations (2) from the left with these zero modes, we are led to the zero-level Lagrangean constraints

$$
\varphi_{a}^{(0)}=\sum_{\alpha} u_{\alpha}^{(0)}(a) \frac{\partial V}{\partial Q_{\alpha}}=0, \quad a=1, \cdots, n_{0}
$$


Some of these constraints may vanish identically. The remaining ones we denote by $\varphi_{a_{0}}^{(0)}$. The corresponding zero modes $u^{(0)}\left(a_{0}\right)$ we refer to as "nontrivial".

In general there are further constraints hidden in equations (2). In order to unravel them, we implement their conservation by adjoining their time derivatives

$$
\left(\frac{\partial \varphi_{a_{0}}^{(0)}}{\partial Q_{\alpha}}\right) \dot{Q}_{\alpha}=0
$$

to the equations (2). This leads to the following enlarged set of equations

$$
W_{A_{1} \beta}^{(1)}(Q) \dot{Q}_{\beta}=K_{A_{1}}^{(1)}(Q)
$$

where $W_{A_{1} \beta}^{(1)}$ are now the elements of a rectangular matrix

$$
W_{A_{1} \beta}^{(1)}:=\left(\begin{array}{c}
W_{\alpha \beta}^{(0)} \\
M_{a_{0} \beta}^{(0)}
\end{array}\right),
$$

with

$$
M_{a_{0} \beta}^{(0)}=\frac{\partial \varphi_{a_{0}}^{(0)}}{\partial Q_{\beta}}
$$

and

$$
K^{(1)}=\left(\begin{array}{c}
\vec{K}^{(0)} \\
\overrightarrow{0}
\end{array}\right)
$$

where

$$
\vec{K}^{(0)}=\vec{\nabla} V(Q)
$$

We now look for "non-trivial" zero modes $\left(u^{(1)}\left(a_{1}\right), a_{1}=1, \cdots, n_{1}\right)$ of $W^{(1)}$, and repeat the steps above, adjoining the time derivative of any new constraints to the equations of motion (6). Repeating this algorithm, the iterative process terminates after $L$ steps, when no new constraints are generated.

Denote the full set of constraints generated by the algorithm collectively by $\left\{\varphi_{a}\right\}, a=1, \cdots, N$. Denote further the set $\{\alpha, a\}$ collectively by $\{A\}$. The final set of equations can then be written in the form

$$
W_{A \beta} \dot{Q}_{\beta}=K_{A}
$$


where

$$
W_{A \beta}:=\left(\begin{array}{c}
W_{\alpha \beta}^{(0)} \\
M_{a \beta}
\end{array}\right),
$$

with

$$
M_{a \beta}=\frac{\partial \varphi_{a}}{\partial Q_{\beta}}
$$

and

$$
K_{A}:=\left(\begin{array}{c}
\vec{K}^{(0)} \\
\overrightarrow{0}
\end{array}\right) .
$$

Denoting by $\vec{u}(a)$ the left zero-modes of the matrix $W_{A \beta}$, the constraints are given by $\varphi_{a}=\vec{u}(a) \cdot \vec{K}=0$.

Equations (11) represent $n+N$ equations for the $n$ velocities $\left\{\dot{Q}_{\alpha}\right\}$. In general such a set of equations would be overdetermined and admit no nontrivial solution. Since the additional $N$ equations were however generated by a self-consistent algorithm from the original Euler-Lagrange equations, the equations (11) do in fact admit a non-trivial solution. In the following we shall assume the first order Lagrangean (1) to describe a purely second class system in the Dirac terminology. In that case we have the following

\section{Assertion:}

The unique solution to (11) for the velocities is given by

$$
\dot{Q}_{\alpha}=F_{\alpha \beta}^{-1} K_{\beta}^{(0)}
$$

where $F^{-1}$ is the inverse of the matrix $F$ obtained by extending the rectangular matrix $W$ defined in (12) to the antisymmetric square matrix

$$
F_{A B}:=\left(\begin{array}{cc}
W_{\alpha \beta}^{(0)} & -M_{\alpha b}^{T} \\
M_{a \beta} & \mathbf{0}
\end{array}\right)
$$

with $M_{a \beta}$ defined in (13), and $M_{\alpha b}^{T}=M_{b \alpha}$.

Proof of Assertion: 
Consider an enlarged space on which the square matrix (16) is to act (we streamline the notation in a self-evident way),

$$
\xi_{A}:=\left(Q_{\alpha}, \rho_{a}\right)
$$

and the following equations:

$$
F_{A B} \dot{\xi}_{B}=K_{A}
$$

As we shall prove further below, $\operatorname{det} F \neq 0$ for a second class system. Hence we can solve these equations for the velocities $\dot{\xi}_{B}$ :

$$
\dot{\xi}_{A}=F_{A B}^{-1} K_{B}
$$

We write the inverse matrix $F^{-1}$ in the form

$$
F_{A B}^{-1}:=\left(\begin{array}{cc}
\tilde{W}_{\alpha \beta}^{(0)} & -\tilde{M}_{\alpha b}^{T} \\
\tilde{M}_{a \beta} & \omega_{a b}
\end{array}\right) .
$$

Then $F^{-1} F=1$ and $F F^{-1}=1$ respectively imply,

$$
\begin{aligned}
& \tilde{W}_{\alpha \gamma}^{(0)} W_{\gamma \beta}^{(0)}-\tilde{M}_{\alpha c} M_{c \beta}=\delta_{\alpha \beta} \\
& \tilde{W}_{\alpha \gamma}^{(0)} M_{\gamma b}^{T}=0 \\
& \tilde{M}_{a \gamma} W_{\gamma \beta}^{(0)}+\omega_{a c} M_{c \beta}=0 \\
& \tilde{M}_{a \gamma} M_{\gamma b}^{T}=-\delta_{a b},
\end{aligned}
$$

and

$$
\begin{aligned}
& W_{\alpha \gamma}^{(0)} \tilde{W}_{\gamma \beta}^{(0)}-M_{\alpha c}^{T} \tilde{M}_{c \beta}=\delta_{\alpha \beta} \\
& \tilde{M}_{a \gamma} \tilde{W}_{\gamma \beta}^{(0)}=0 \\
& W_{\alpha \gamma}^{(0)} \tilde{M}_{\gamma b}^{T}+M_{\alpha c}^{T} \omega_{c b}=0 \\
& M_{a \gamma} \tilde{M}_{\gamma b}^{T}=-\delta_{a b} .
\end{aligned}
$$

Consider eqs. (18) which, written out explictely, read

$$
W_{\alpha \beta}^{(0)} \dot{Q}_{\beta}-M_{\alpha b}^{T} \dot{\rho}_{b}=K_{\alpha}^{(0)}
$$




$$
M_{a \beta} \dot{Q}_{\beta}=0
$$

From (13) we see that the last equation states that $\dot{\varphi}_{a}=0$, where $\varphi_{a}=0$ are the constraints hidden in the equations of motion (21). Because of this, requiring their presistance in time implies that the second term on the LHS of (25) must vanish. Making use of (22) this in turn implies that $\dot{\rho}_{a}=0$ for all $a$. Pl Setting $\dot{\rho}_{a}=0$ in (19), we have from (20),

$$
\begin{gathered}
\dot{Q}_{\alpha}=\tilde{W}_{\alpha \beta}^{(0)} K_{\beta}^{(0)} \\
0=\tilde{M}_{a \beta} K_{\beta}^{(0)}
\end{gathered}
$$

Eq. (28) is just the statement that $\varphi_{a}=0$. To see this we notice that according to (21), the vectors

$$
\vec{u}_{A}(a):=\left(\tilde{M}_{a \gamma}, \omega_{a c}\right)
$$

are just the left zero modes of the matrix (12). Hence

$$
\tilde{M}_{a \beta} K_{\beta}^{(0)}=u_{A}(a) K_{A}=\varphi_{a} .
$$

As we now show, eqs. (19) are nothing but the Hamilton equations of motion derived from the so-called extended Hamiltonian. By making contact with the Hamiltonian formalism, we will prove that i) $F$ is an invertible matrix, and ii) the solutions to (25) imply that $\dot{\rho}_{a}=0$, as was claimed above. This, at the same time, will prove the uniqueness of the solution.

From the Dirac point of view, the symplectic Lagrangean (四) descibes a system with a primary constraint for every coordinate $Q_{\alpha}$ :

$$
\phi_{\alpha}:=P_{\alpha}-a_{\alpha}(Q)=0, \quad \alpha=1, \cdots, n
$$

where $P_{\alpha}$ are the momenta canonically conjugate to the coordinates $Q_{\alpha}$. Since the Lagrangean (1) is first order in the time derivatives, the corresponding canonical Hamiltonian $H_{c}$ is just given by the potential $V$,

$$
H_{c}=V(Q)
$$

\footnotetext{
${ }^{3}$ An alternative proof that this must indeed be the case will be given further below,
} where we make contact with the Hamiltonian formalism. 
and hence does not depend on the momenta. The dependence on the momenta enters only in the total Hamiltonian via the primary constraints:

$$
H_{T}(Q, P)=V(Q)+\sum_{\alpha} v_{\alpha} \phi_{\alpha}(Q, P)
$$

The Dirac algorithm will in general lead to secondary constraints, which we label by a latin index: $\varphi_{a}=0$. It is easy to see that they are identical with the constraints generated by the Lagrangean algorithm. Thus consider the persistance equations for the primary constraints $\phi_{\alpha}$ :

$$
\left\{\phi_{\alpha}, H_{T}\right\}=\left\{\phi_{\alpha}, V\right\}+\sum_{\beta}\left\{\phi_{\alpha}, \phi_{\beta}\right\} v_{\beta}=0
$$

From (31) and (3) we see that $\left\{\phi_{\alpha}, \phi_{\beta}\right\}=W_{\alpha \beta}^{(0)}(Q)$, so that the above eqiations read,

$$
W_{\alpha \beta}^{(0)} v_{\beta}=K_{\alpha}^{(0)}
$$

Multiplying this equation with the left-zero modes of $W^{(0)}$ we arrive at the level-zero Lagrangean constraints (4), which are only functions of $Q$. Requiring their persistance in time as generated by $H_{T}$ yields $M_{a \beta}^{(0)} v_{\beta}=0$, and adjoining these equations to (34),

$$
W_{A_{1} \beta}^{(1)} v_{\beta}=K_{A_{1}}^{(1)}
$$

By taking appropriate linear combinations of these equations, new constraints may be generated which are functions of only the $Q_{\alpha}$ 's. This just corresponds to looking for left-zero modes of $W^{(1)}$. The new constraints are thus identical with those derived in the Lagrangean approach at level "one". Proceeding in this way it is easy to see that the secondary constraints generated by the Dirac algorithm applied to $H_{T}(Q, P)$ are identical with the constraints $\left\{\varphi_{a}=0\right\}$ generated by the Lagrangean algorithm.

We now go over to the extended Hamiltonian by including the secondary constraints with their respective Lagrange multipliers $\bar{v}_{a}$,

$$
H_{T} \rightarrow H_{E}=H_{c}+\sum_{B} \lambda_{B} \Omega_{B}
$$


where

$$
\Omega_{A}:=\left(\phi_{\alpha}, \varphi_{a}\right), \quad \lambda_{A}:=\left(v_{\alpha}, \bar{v}_{a}\right)
$$

The Hamilton equations of motion for the coordinates $Q_{\alpha}$ associated with the extended Hamiltonian $H_{E}$, read

$$
\begin{gathered}
\dot{Q}_{\alpha}=\left\{Q_{\alpha}, H_{E}\right\}=v_{\alpha}, \\
\dot{P}_{\alpha}=\left\{P_{\alpha}, H_{E}\right\}=-K_{\alpha}^{(0)}+v_{\beta} \partial_{\alpha} a_{\beta}-\bar{v}_{b} \partial_{\alpha} \varphi_{b}
\end{gathered}
$$

Consistency with the persistance in time of the primary constraints requires

$$
\dot{\phi}_{\alpha}=\dot{P}_{\alpha}-\dot{a}_{\alpha}=0
$$

One readily verifies from (37) that this leads to

$$
W_{\alpha \beta}^{(0)} v_{\beta}-M_{\alpha b}^{T} \bar{v}_{b}=K_{\alpha}^{(0)}
$$

On the other hand, persistance of the secondary constraints $\varphi_{a}$ leads to

$$
M_{b \beta} v_{\beta}=0
$$

Upon making use of (36), we thus retrieved equations (25) and (26) if we identify $\bar{v}_{a}$ with $\dot{\rho}_{a}$. Hence in the Hamiltonian formalism these equations are merely the persistance equations of the primary and secondary constraints, which can be compactly written in the Hamiltonian form

$$
\left\{\Omega_{A}, H_{c}\right\}+\sum_{B}\left\{\Omega_{A}, \Omega_{B}\right\} \lambda_{B}=0
$$

We now recognize that the matrix elements of $F$ in (16) are given by

$$
F_{A B}=\left\{\Omega_{A}, \Omega_{B}\right\}
$$

Since the constraints have been assumed to be second class, this matrix is invertible. Noting further that

$$
\left\{\Omega_{A}, H_{c}\right\}=-K_{A},
$$


it follows from (41) that

$$
\lambda_{A}=F_{A B}^{-1} K_{B}
$$

With $\dot{Q}_{\alpha}=v_{\alpha}$, these equations are nothing but (19), with $\dot{\rho}_{a}$ identified with $\bar{v}_{a}$

To prove the equivalence of (39) and (40) with the original set of equations (11), we must still show that eqs. (42) imply that $\bar{v}_{a}=0$. To this effect we recall that the secondary constraints $\varphi_{a}=0$ have actually been generated by the total Hamiltonian $H_{T}$ from the persistance equations

$$
\left\{\Omega_{A}, H_{c}\right\}+\sum_{\beta}\left\{\Omega_{A}, \phi_{\beta}\right\} \lambda_{\beta}=0
$$

Consistency with (41) therefore requires that

$$
\sum_{b}\left\{\Omega_{A}, \varphi_{b}\right\} \bar{v}_{b}=0
$$

or equivalently $M_{\alpha b}^{T} \bar{v}_{b}=0$, which, upon making use of (22), implies $\bar{v}_{a}=0$. This completes the proof of our assertion.

Concluding this section we have therefore shown the full equivalence of the Lagrangean and Hamiltonian approach to the theory described by the first order Lagrangean (11). Any other approach must therefore reproduce the constrained structure of the Lagrangean approach. An alternative (symplectic) algorithm for unravelling the constrained structure was proposed in ref. [4]. In the following section we will show that the symplectic algorithm does not necessarily generate the correct constrained structure.

\section{The symplectic algorithm}

In the following we first illustrate in terms of a simple example, an alternative algorithm for generating the constraints, as proposed in ref. [4]. We refer to it as the "symplectic algorithm". 


\subsection{Particle on a Hypersphere}

The following (second order) Lagrangean is referred to as describing the nonlinear sigma model in Quantum Mechanics:

$$
L=\frac{1}{2} \cdot \vec{q}^{2}+\lambda\left(\vec{q}^{2}-1\right)
$$

where $\vec{q}=\left(q_{1}, \cdots, q_{n}\right)$. The equivalent symplectic Lagrangean reads,

$$
L^{(0)}=\vec{p} \cdot \overrightarrow{\dot{q}}-V^{(0)}
$$

with

$$
V^{(0)}=-\lambda\left(\vec{q}^{2}-1\right)+\frac{1}{2} \vec{p}^{2}
$$

The Lagrangean is of the form

$$
L^{(0)}=a_{\alpha}(Q) \dot{Q}_{\alpha}-V^{(0)}(Q)
$$

with

$$
Q_{\alpha}:=(\vec{q}, \vec{p}, \lambda), \quad a_{\alpha}:=(\vec{p}, \overrightarrow{0}, 0)
$$

The equations of motion are of the form (2), with

$$
W^{(0)}=\left(\begin{array}{ccc}
\mathbf{0} & -\mathbf{1} & \overrightarrow{0} \\
\mathbf{1} & \mathbf{0} & \overrightarrow{0} \\
\overrightarrow{0}^{T} & \overrightarrow{0}^{T} & 0
\end{array}\right)
$$

and

$$
K_{\alpha}^{(0)}=\left(\begin{array}{c}
-2 \lambda \vec{q} \\
\vec{p} \\
-\left(\vec{q}^{2}-1\right)
\end{array}\right)
$$

The matrix $W^{(0)}$ has one "zero-level" zero mode:

$$
u_{\alpha}^{(0)}:=(\overrightarrow{0}, \overrightarrow{0}, 1)
$$

implying the constraint

$$
\varphi^{(0)}=-u_{\alpha}^{(0)} K_{\alpha}^{(0)}=\vec{q}^{2}-1=0
$$


This constraint will necessarily coincide with that of the Lagrangean approach at the zero'th level.

In the symplectic algorithm the time derivative of the constraint (50) is however added in the (partially integrated) form $-\dot{\rho}^{(0)} \varphi^{(0)}$ to the zero-level Lagrangean (46), It to yield the first level Lagrangean

$$
L^{(1)}=L^{(0)}-\dot{\rho}^{(0)} \varphi^{(0)},
$$

where $\rho^{(0)}$ is a new dynamical variable. Correspondingly we define the extended set of coordinates

$$
\xi_{A_{1}}^{(1)}:=\left(\vec{q}, \vec{p}, \lambda, \rho^{(0)}\right) .
$$

$L^{(1)}$ can be written in the form

$$
L^{(1)}=a_{A_{1}}^{(1)} \dot{\xi}_{A_{1}}^{(1)}-V^{(0)}(Q),
$$

where

$$
a_{A_{1}}^{(1)}:=\left(\vec{p}, \overrightarrow{0}, 0,-\varphi^{(0)}\right) .
$$

The corresponding Euler-Lagrange equations read

$$
F_{A_{1} B_{1}}^{(1)} \dot{\xi}_{B_{1}}^{(1)}=K_{A_{1}}^{(1)}
$$

where the "first-level" symplectic square matrix is given by,

$$
F^{(1)}=\left(\begin{array}{cccc}
\mathbf{0} & \mathbf{- 1} & \overrightarrow{0} & -2 \vec{q} \\
\mathbf{1} & \mathbf{0} & \overrightarrow{0} & \overrightarrow{0} \\
\overrightarrow{0}^{T} & \overrightarrow{0}^{T} & 0 & 0 \\
2 \vec{q}^{T} & \overrightarrow{0}^{T} & 0 & 0
\end{array}\right)
$$

and

$$
K^{(1)}=\left(\begin{array}{c}
-2 \lambda \vec{q} \\
\vec{p} \\
-\left(\vec{q}^{2}-1\right) \\
0
\end{array}\right) .
$$

\footnotetext{
${ }^{4}$ In ref. [4] the term proportional to the Lagrange multiplier $\lambda$ in $L^{(0)}$ has been absorbed into the term proportional to $\dot{\rho}$.
} 
$F^{(1)}$ has two (level-one) zero modes,

$$
\begin{gathered}
u_{A_{1}}^{(1)}(1):=(\overrightarrow{0}, \overrightarrow{0}, 1,0) \\
u_{A_{1}}^{(1)}(2):=(\overrightarrow{0}, 2 \vec{q}, 0,-1)
\end{gathered}
$$

The first zero mode reproduces the constraint $\vec{q}^{2}-1=0$. The second zero mode yields the new constraint

$$
\varphi^{(1)}=\vec{u}^{(1)}(2) \cdot \vec{K}^{(1)}=2 \vec{p} \cdot \vec{q}
$$

As one easily verifies, these constraints are identical with those obtained in the Lagrangean algorithm described in section 1, at this level.

According to the symplectic algorithm we now define the second level Lagrangean by adding the new constraint in the form

$$
L^{(2)}=L^{(0)}-\dot{\rho}^{(0)} \varphi^{(0)}-\dot{\rho}^{(1)} \varphi^{(1)}
$$

or

$$
L^{(2)}=a_{A_{2}}^{(2)} \dot{\xi}_{A_{2}}^{(2)}-V^{(0)}(Q)
$$

with

$$
\xi_{A_{2}}^{(2)}:=\left(\vec{q}, \vec{p}, \lambda, \rho^{(0)}, \rho^{(1)}\right)
$$

and

$$
a_{A_{2}}^{(2)}:=\left(\vec{p}, \overrightarrow{0}, 0,-\left(\vec{q}^{2}-1\right),-2 \vec{p} \cdot \vec{q}\right)
$$

For the corresponding symplectic matrix one obtains

$$
F^{(2)}=\left(\begin{array}{ccccc}
\mathbf{0} & -\mathbf{1} & \overrightarrow{0} & -2 \vec{q} & -2 \vec{p} \\
\mathbf{1} & \mathbf{0} & \overrightarrow{0} & \overrightarrow{0} & -2 \vec{q} \\
\overrightarrow{0}^{T} & \overrightarrow{0}^{T} & 0 & 0 & 0 \\
2 \vec{q}^{T} & \overrightarrow{0}^{T} & 0 & 0 & 0 \\
2 \vec{p}^{T} & 2 \vec{q}^{T} & 0 & 0 & 0
\end{array}\right)
$$

As one readily checks, this matrix has only one zero mode $u_{A_{2}}^{(2)}:=\left(\overrightarrow{0}^{T}, \overrightarrow{0}^{T}, 1,0,0\right)$ which, however, just reproduces the constraint $\varphi^{(0)}=0$. Hence the symplectic algorithm terminates at this point, leaving us with a non-invertible 
matrix. I On the other hand, one readily checks that the standard Lagrangean (or equivalently, Dirac) algorithm generates not only the constraints $\vec{q}^{2}-1=0, \vec{p} \cdot \vec{q}=0$, but also one futher constraint $2 \lambda \vec{q}^{2}+\vec{p}^{2}=0$. Indeed, in the Lagragian algorithm, $F^{(2)}$ in (54) is replaced by the rectangular matrix

$$
W^{(2)}=\left(\begin{array}{ccc}
\mathbf{0} & -\mathbf{1} & \overrightarrow{0} \\
\mathbf{1} & \mathbf{0} & \overrightarrow{0} \\
\overrightarrow{0}^{T} & \overrightarrow{0}^{T} & 0 \\
2 \vec{q}^{T} & \overrightarrow{0}^{T} & 0 \\
2 \vec{p}^{T} & 2 \vec{q}^{T} & 0
\end{array}\right)
$$

which is seen to possess the three zero modes,

$$
u^{(2)}(1)=\left(\begin{array}{c}
\overrightarrow{0} \\
\overrightarrow{0} \\
1 \\
0 \\
0
\end{array}\right),, \quad u^{(2)}(2)=\left(\begin{array}{c}
0 \\
2 \vec{q} \\
0 \\
-1 \\
0
\end{array}\right), \quad u^{(2)}(3)=\left(\begin{array}{c}
2 \vec{q} \\
-2 \vec{p} \\
0 \\
0 \\
1
\end{array}\right),
$$

which in addition to the constraints $\varphi^{(0)}=0, \varphi^{(1)}=0$, imply a new constraint

$$
\varphi^{(2)}:=2 \lambda \vec{q}^{2}+\vec{p}^{2}=0
$$

Hence we are taken to a third level with the corresponding enlarged matrix given by

$$
W^{(3)}=\left(\begin{array}{ccc}
\mathbf{0} & \mathbf{- 1} & \overrightarrow{0} \\
\mathbf{1} & \mathbf{0} & \overrightarrow{0} \\
\overrightarrow{0}^{T} & \overrightarrow{0}^{T} & 0 \\
2 \vec{q}^{T} & \overrightarrow{0}^{T} & 0 \\
2 \vec{p}^{T} & 2 \vec{q}^{T} & 0 \\
4 \lambda \vec{q}^{T} & 2 \vec{p}^{T} & 2 \vec{q}^{2}
\end{array}\right) .
$$

\footnotetext{
${ }^{5}$ In ref. Theirby the information about $\lambda$ was lost, and the resulting matrix $F^{(2)}$ at level 2 became invertible.
} 
As one readily checks, $W^{(3)}$ has no new zero modes. Hence the algorithm terminates at this point. Notice that the extension of this matrix to a square matrix as discussed in section 2 results in an invertible matrix, reflecting a second class system.

We see that the symplectic algorithm fails to generate the correct set of constraints known to be present for the model in question. In fact, from the point of view of the second order Lagrangean formulation there exists just one primary constraint $\phi=p_{\lambda}=0$, where $p_{\lambda}$ is the momentum conjugate to the variable $\lambda$, and the total Hamiltonian correspondingly reads, $H_{T}=$ $\frac{1}{2} \vec{p}^{2}-\lambda\left(\vec{q}^{2}-1\right)+v p_{\lambda}$. As one readily checks, the last constraint (57) just serves to fix the Lagrange multiplier $v$ in $H_{T}$ to $v=0$. Only at this final stage the second class nature of the model in question becomes evident. If we stop at level two, $v$ remains arbitrary, as expressed by the zero column in (54) and (55).

\subsection{When does the symplectic algorithm fail?}

We now examine in general terms at which point the symplectic algorithm begins to fail. To this end we examine what the symplectic algorithm described above corresponds to on Hamiltonian level. Let $L^{(0)}$ be of the form (1), with (31) the corresponding primary constraints. At the $\ell+1$ 'th level, the symplectic algorithm leads to a Lagrangean of the form (we streamline the notation)

$$
L^{(\ell+1)}=L^{(0)}-\sum_{a_{\ell}} \dot{\rho}_{a_{\ell}} \varphi_{a_{\ell}}(Q),
$$

where $\varphi_{a_{\ell}}, a_{\ell}=1, \cdots, n_{\ell}$ denote all the constraints generated by the iterative procedure up to level $\ell$. The corresponding total Hamiltonian reads,

$$
H_{T}^{(\ell+1)}=H_{T}^{(0)}+\sum_{a_{\ell}} \lambda_{a_{\ell}} \phi_{a_{\ell}}
$$


Here $\left\{\phi_{a_{\ell}}\right\}$ denote the corresponding set of primary constraints associated with $\left\{\dot{\rho}_{a_{\ell}}\right\}$,

$$
\phi_{a_{\ell}}=P_{a_{\ell}}+\varphi_{a_{\ell}}(Q)
$$

where $P_{a_{\ell}}$ are the momenta conjugate to the dynamical variables $\rho_{a_{\ell}}$ and

$$
H_{T}^{(0)}=H^{(0)}+\sum_{\alpha} v_{\alpha} \phi_{\alpha}
$$

with $\phi_{\alpha}$ the primary constraints (31), associated with the original Lagrangean $L^{(0)}$. Hence in the symplectic algorithm described above, the total Hamiltonian is modified at each level. Clearly the Euler-Lagrange equations derived from $L^{(\ell+1)}$ and the Hamilton equations of motion following from $H^{(\ell+1)}$ describe the same dynamics.

Conservation of all the primary constraints requires,

$$
\begin{aligned}
\left\{\phi_{\alpha}, H_{T}^{(\ell+1)}\right\} & =-\frac{\partial V}{\partial Q_{\alpha}}+\sum_{\beta}\left\{\phi_{\alpha}, \phi_{\beta}\right\} v_{\beta}+\sum_{b_{\ell}}\left\{\phi_{\alpha}, \phi_{b_{\ell}}\right\} \lambda_{b_{\ell}}=0 \\
\left\{\phi_{a_{\ell}}, H_{T}^{(\ell+1)}\right\} & =\sum_{\beta}\left\{\phi_{a_{\ell}}, \phi_{\beta}\right\} v_{\beta}+\sum_{b_{\ell}}\left\{\phi_{a_{\ell}}, \phi_{b_{\ell}}\right\} \lambda_{b_{\ell}}=0 .
\end{aligned}
$$

Let $\Phi_{A_{\ell}}$ stand for

$$
\Phi_{A_{\ell}}:=\left(\phi_{\alpha}, \phi_{a_{\ell}}\right)
$$

with $\lambda_{A_{\ell}}$ the corresponding set of Lagrange multipliers:

$$
\lambda_{A_{\ell}}:=\left(v_{\alpha}, \lambda_{a_{\ell}}\right)
$$

Then we may write (59) in the compact form

$$
\sum_{B_{\ell}}\left\{\Omega_{A_{\ell}}, \Omega_{B_{\ell}}\right\} \lambda_{B_{\ell}}=K_{A_{\ell}}
$$

where

$$
\vec{K}=(\vec{\nabla} V, \overrightarrow{0})
$$

with $\overrightarrow{0}$ an $N_{\ell}=n+n_{\ell}$ - component null-vector.

One readily checks that $\left\{\Omega_{A_{\ell}}, \Omega_{B_{\ell}}\right\}$ is identical with $F_{A_{\ell} B_{\ell}}$ in (16) at the $\ell$ 'th level. Furthermore, with the identification of $v_{\alpha}$ and $\lambda_{a_{\ell}}$ with $\dot{Q}_{\alpha}$ and 
$\dot{\rho}_{a_{\ell}}$ via the Hamilton equations of motion,

$$
\begin{aligned}
& \dot{Q}_{\alpha}=\left\{Q_{\alpha}, H_{T}^{(\ell+1)}\right\}=v_{\alpha}, \\
& \dot{\rho}_{a_{\ell}}=\left\{\rho_{a_{\ell}}, H_{T}^{(\ell+1)}\right\}=\lambda_{a_{\ell}}
\end{aligned}
$$

we see that the persistance equations (59) are just the equations of motion obtained from $L^{(\ell+1)}$ in the symplectic approach.

Within the Hamiltonian formalism, the search for zero modes of $F$ at level $\ell$ now corresponds to seeking linear combinations of all the primaries, $u_{A_{\ell}} \Phi_{A_{\ell}}$, such that

$$
\sum_{A_{\ell}} u_{A_{\ell}}\left\{\Phi_{A_{\ell}}, \Phi_{B_{\ell}}\right\}=0
$$

From (60) we see that these equations imply linearly independent (nontrivial) constraints, which we denote by

$$
\varphi_{a_{\ell}}=\sum u_{A_{\ell}}\left(a_{\ell}\right) K_{A_{\ell}}=0
$$

Of the conditions (61), only those with $B_{\ell}=\beta$,

$$
\sum_{A_{\ell}} u_{A_{\ell}}\left(a_{\ell}\right)\left\{\Phi_{A_{\ell}}, \phi_{\beta}\right\}=0
$$

are contained in the Lagrangean (and hence traditional Dirac) approach. Let $u\left(a_{\ell}\right)$ be solutions of (62). From (61), with $B_{\ell}=b_{\ell}$ we see that the symplectic approach thus implies the additional restrictions

$$
u_{\alpha}\left(a_{\ell}\right) \frac{\partial \varphi_{b_{\ell}}}{\partial Q_{\alpha}}=0
$$

for the zero modes, which are not contained in the Lagrangean algorithm. Hence we have a mismatch between the symplectic and Lagrangean algorithm, once the latter condition is not satisfied in the iterative process, and the constraint structure becomes inequivalent for the two algorithms. This is the main point of this paper. 6

\footnotetext{
${ }^{6}$ This subtle point has been missed in ref. 10
} 
Let us exemplify this for the case of the "particle on a hypersphere". At the second level the Lagrangean algorithm leads to the zero modes (56). We verify that at the zero'th and first level of the iterative process the condition (63) is still verified, whereas this is not the case for the second level zero mode $u^{(2)}(3)$ in $(56)$, since

$$
u_{\alpha}^{(2)}(3) \frac{\partial \varphi^{(0)}}{\partial Q_{\alpha}}=4 \vec{q}^{2} \neq 0 .
$$

This explains why the algorithm stops before generating one further constraint, $\varphi^{(2)}=0$, eq. (57).

It is instructive to further ellucidate the meaning of this finding. Going through the iterative procedure on Hamiltonian level (found above to be equivalent to the symplectic algorithm), we arrive after the second iterative step at the Hamiltonian

$$
\begin{aligned}
H_{T}^{(2)} & =V^{(0)}(q, p, \lambda)+\sum_{i=1}^{n}\left(v_{q_{i}}\left(P_{q_{i}}-p_{i}\right)+v_{p_{i}} P_{p_{i}}\right) \\
& +v p_{\lambda}+\lambda_{1}\left(P_{1}+\vec{q}^{2}-1\right)+\lambda_{2}\left(P_{2}+2 \vec{q} \cdot \vec{p}\right)
\end{aligned}
$$

Conservation in time of the primaries now merely serves to fix all the Lagrange multipliers $\lambda, \lambda_{1}, \lambda_{2}, \lambda_{2}$, and leads to:

$$
\begin{aligned}
\vec{v}_{p}+2 \lambda_{1} \vec{q}+2 \lambda_{2} \vec{p} & =2 \lambda \vec{q} \\
\vec{v}_{q}+2 \lambda_{2} \vec{q} & =\vec{p} \\
\vec{v}_{q} \cdot \vec{p}+\vec{v}_{p} \cdot \vec{q} & =0 \\
\vec{v}_{q} \cdot \vec{q} & =0,
\end{aligned}
$$

as well as the constraint $\vec{q}^{2}-1=0$. These equations may be solved for $\lambda_{1}$ and $\lambda_{2}$,

$$
\begin{aligned}
& \lambda_{1}=\frac{1}{2}\left(\vec{p}^{2}+2 \lambda-2 \vec{p} \cdot \vec{q}\right) \\
& \lambda_{2}=\frac{1}{2}(\vec{p} \cdot \vec{q}),
\end{aligned}
$$

and hence vor $v_{q_{i}}$ and $v_{p_{i}}$, leaving $v$ undetermined. Hence $\vec{q}^{2}-1=0$ is the only constraint (as reflected by the zero column in (54) and (55)), unless we 
set $\lambda_{1}=\lambda_{2}=0$. This just corresponds to working with the total Hamiltonian

$H_{T}^{(0)}$. In that case the algorithm does not terminate, but rather generates one further constraint, $\vec{p}^{2}+2 \lambda=0$, whose time independence will finally fix also $v$ to vanish.

\section{Conclusion}

In this paper we have examined the interrelation between three different algorithms currently in use for unravelling the constrained structure of first order Langrangians. We have referred to these as the "Lagrangean", "Dirac" and "symplectic" algorithms. Of these the first two rest on a solid foundation, and, as we have seen, there exists a one-to-one correspondance between these formalisms. In particular we have shown how to invert the seemingly overdetermined system of equations of the Lagrangean algorithm. As for the symplectic algorithm presented in refs. [4, it does not always reproduce the correct set of constraints, as we have seen. In fact, we have shown for a general first order Lagrangean, under what conditions the algorithm fails to reproduce all of the constraints correctly. A concrete example has exemplified this.

\section{References}

[1] P. A. M. Dirac, Lectures on quantum mechanics (Belfer graduate School, Yeshiba University Press, New York, 1964 ).

[2] L. Faddeev and R. Jackiw, Phys. Rev. Lett. 60, 1692 (1988). Phys. Rev. D49, 5438 (1994); Nucl. Phys. B426, 129 (1994); Phys. Rev. D55, 6339 (1999).

[3] A. Shirzad, J. Phys. A: ,Math.Gen 312747 (1998). R. Banerjee, H. J. Rothe and K. D. Rothe, Phys. Lett. B463, 248 (1999); ibid., B479, 
429 (2000), J. Phys. A: Math.Gen 332059 (2000). X. Gracia and J.M. Pons, Ann.Phys. 187, 355 (1988). H.J. Rothe, Lagrangean approach to Hamiltonian symmetries and the Dirac conjecture, hep-th/0205243

[4] J. Barcelos-Neto and C. Wotzasek, Mod. Phys. Lett. A7, 1172 (1992); Int. J. Mod. Phys. A7, 4981 (1992); H. Montani and C. Wotzasek, Mod. Phys. Lett. A8, 3387 (1993); H. Montani, Int. J. Mod. Phys. A8, 3419 (1993).

[5] R. Banerjee and J. Barcelos-Neto, Nucl. Phys. B499,

[6] Y.W. Kim, Y.J. Park, K.Y. Kim, Y. Kim, and C.H. Kim, J. Korean Phys. Soc. 26, 243 (1993); Y.W. Kim, Y.J. Park, K.Y. Kim, and Y. Kim, J. Korean Phys. Soc. 27, 610 (1994).

[7] S.K. Kim, Y.W. Kim, Y.J. Park, Y. Kim, C.H. Kim and W.T. Kim, J. Korean Phys. Soc. 28, 128 (1995)

[8] Soon-Tae Hong, Yong-Wan Kim, Young-Jai Park and K.D. Rothe, Mod. Phys. Lett. A17, 435 (2002).

[9] Soon-Tae Hong, Yong-Wan Kim, Young-Jai Park and K.D. Rothe, hepth/01112170.

[10] A. Shirzad and M. Mojiri, Mod. Phys. Lett. A16,2439 (2001). 